\title{
Stay mindfully active during the coronavirus pandemic: a feasibility study of mHealth-delivered mindfulness yoga program for people with Parkinson's disease
}

\author{
Jojo Yan Yan Kwok ${ }^{1^{*}} \mathbb{0}$, Jung Jae Lee ${ }^{1} \mathbb{0}$, Edmond Pui Hang Choi ${ }^{1} \mathbb{0}$, Pui Hing Chau ${ }^{1} \mathbb{0}$ and Man Auyeung ${ }^{2}$
}

\begin{abstract}
Importance: Patients with long-term neurological conditions, such as Parkinson's disease (PD), are particularly vulnerable to the public health measures taken to combat the COVID-19 pandemic. The inaccessibility of center-based rehabilitation further aggravated their motor dysfunctions as well as mental distress, leading to exacerbation of motor and non-motor symptoms, high healthcare utilization and worsened health-related quality of life (HRQOL).

Objective: This study aimed to evaluate the feasibility, safety, and preliminary effects of the mHealth-delivered home-based mindfulness yoga program on functional balance, motor symptoms, mental health and HRQOL in patients with PD.
\end{abstract}

Design, setting and participants: This prospective, single-arm, non-randomized feasibility study adopted a sequential explanatory mixed-method design. Adults (aged $\geq 18$ ) with a clinical diagnosis of idiopathic Parkinson's disease (Hoehn and Yahr stage I to III) who were able to stand unaided and walk with or without an assistive device were enrolled via convenience sampling.

Intervention: Home-based mindfulness yoga training were delivered via video-conferencing software (Zoom) in eight bi-weekly 90-min sessions.

Main outcomes and measures: This current study measured functional balance, motor symptoms, perceived balance confidence, perceived freezing of gait symptoms, anxiety and depression, mindfulness and HRQOL using a tele-assessment approach at baseline and 1-week post-intervention. All participants were invited to attend qualitative individual interviews to explore their experience of using online mindfulness yoga program as a lifestyle intervention for PD rehabilitation.

Results: Among the ten patients, $80 \%$ completed the program with an adherence rate of $98.4 \%$. All participants were able to learn and practice mindfulness yoga following the eight bi-weekly online mindfulness yoga training sessions, without any significant adverse events. Tele-assessment of outcomes were feasible and uneventful. Qualitative feedback revealed participants had a high preference of using the tele-rehabilitation approach to stay mindful and being active, both physically and socially, while confronting the changes brought by COVID-19 pandemic.

\footnotetext{
*Correspondence: jojoyyk@hku.hk; jojo.yykwok@gmail.com

${ }^{1}$ School of Nursing, Li Ka Shing Faculty of Medicine, The University

of Hong Kong, Hong Kong 4/F, William MW Mong Block, 21 Sassoon

Road, Pokfulam, Hong Kong SAR, China

Full list of author information is available at the end of the article
}

(c) The Author(s) 2022. Open Access This article is licensed under a Creative Commons Attribution 4.0 International License, which permits use, sharing, adaptation, distribution and reproduction in any medium or format, as long as you give appropriate credit to the original author(s) and the source, provide a link to the Creative Commons licence, and indicate if changes were made. The images or other third party material in this article are included in the article's Creative Commons licence, unless indicated otherwise in a credit line to the material. If material is not included in the article's Creative Commons licence and your intended use is not permitted by statutory regulation or exceeds the permitted use, you will need to obtain permission directly from the copyright holder. To view a copy of this licence, visit http://creativecommons.org/licenses/by/4.0/. The Creative Commons Public Domain Dedication waiver (http://creativeco mmons.org/publicdomain/zero/1.0/) applies to the data made available in this article, unless otherwise stated in a credit line to the data. 
Conclusions and relevance: The mHealth-delivered home-based mindfulness yoga intervention was feasible, safe, and well-accepted among people with PD to relieve the burden brought by COVID-19 pandemic. Future studies should adopt a design with enhanced rigor, a comparison group, and enlarged sample size to evaluate the efficacy of the program in patients with long-term neurological conditions and/or physical impairments. We recommend a longer intervention duration of at least 8 weeks to enhance the psychophysiological effects.

Keywords: Parkinson's disease, Rehabilitation, Quality of life, Physical exercise, Psychological support, Symptom management, mHealth, Yoga, Mindfulness, Home-based

\section{Background}

Between $47 \%-80 \%$ of people with Parkinson's disease (PD) experience freezing of gait (FOG) [1], which is associated with impaired balance and mobility, falls, fallrelated physical injuries and fractures, functional restriction, increased institutionalization risk, and mortality [2, 3]. Clinical guidelines and previous studies recommend a complementary mind-body approach to PD rehabilitation [4], including yoga, Tai Chi, and dance on improving the motor symptoms and HRQOL of patients with PD [5-8]. However, most programs are delivered face-toface in center-based settings, which are often inaccessible to many patients due to resource limitations, distance, disability, and financial concerns while posing potential health risks in the long run.

For people with PD, exercise is a vital component for maintaining balance, mobility and activities of daily living. With the public health and virus-control measures taken to combat the current coronavirus disease 2019 (COVID-19) pandemic, such as self-quarantine, social distancing, restriction on group gatherings, and community center and gym facility closures, patients with PD are particularly vulnerable due to the reduced accessibility of exercise-related facilities. Many patients spend increased time at home with reduced physical activity; this situation subsequently exacerbates deleterious health consequences, such as physical and functional decline, emotional distress, comorbid conditions, and worsened HRQOL [9-11]. Hence, additional effort should be expended to test and translate evidence- and centerbased rehabilitation practices into home-based settings to address the challenges imposed by the common barriers to participation in center-based rehabilitation and any future infectious disease outbreaks.

Indeed, effective delivery and adherence to rehabilitation programs are of high priority in PD care. In recent years, advances in technologies have provided new avenues for medical and public health practice. For example, mobile devices, such as smartphones, tablets, and laptops (mHealth), have been utilized for chronic illness care. The adoption of mHealth technology offers a promising platform for efficient and accessible intervention delivery [12]. Several studies have demonstrated the efficacy of mHealth interventions for improving the overall HRQOL of patients with chronic illnesses, such as chronic pain, cancer, generalized anxiety disorder and cardiovascular diseases [13, 14]. However, mHealthbased interventions for improving motor symptoms, particularly balance, mobility, and FOG symptoms, as well as mental health and HRQOL, in patients with PD have been limited. The delivery of exercise programs by using a mHealth approach may be ideal for reaching and supporting patients with PD to improve their chronic illness care during infectious disease pandemics, relieve mental distress, and help them stay active at home. Therefore, we further tailored a mindfulness yoga programme, which was delivered interactively through an online platform for PD rehabilitation. This feasibility study aimed to examine the acceptability, safety and implementation of this mHealth-delivered home-based mindfulness yoga program. To our knowledge, this is the first feasibility study that solely used a telemedicine approach to deliver mindfulness yoga intervention to enhance the functional balance, motor symptoms, mental health and HRQOL of patients with PD.

\section{Methods}

\section{Study design}

This prospective, single-armed, non-randomized feasibility study adopted a sequential explanatory mixedmethods design to examine the acceptability, safety, and implementation of a mHealth-delivered home-based mindfulness yoga program for PD patients. The study was approved by the corresponding ethics committee (HKU/HA HKW IRB: UW 19-535) in accordance with the Declaration of Helsinki.

\section{Study participants}

Using convenience sampling, we recruited potential participants through a regional neurology outpatient clinic in Hong Kong from May 2020 to June 2020. Inclusion criteria are: (i) participants who had a clinical diagnosis of idiopathic PD with a disease severity rating of stage I-III on the Hoehn and Yahr scale (rated on a scale of I-V with high numbers indicating severe disease), (ii) were older than 18 years, (iii) able to stand unaided 
for $\geq 10 \mathrm{~min}$, (iv) able to walk with/without any assistive device for $\geq 100 \mathrm{~m}$, (v) have access to the Internet and any mobile device, such as a smartphone, tablet, and laptop, (vi) were willing to download Zoom as the training platform, and (vii) able to give written consent. Exclusion criteria are: (i) those who had been regularly practicing instructor-led mind-body interventions $\geq$ once a week during the past 6 months, (ii) were currently participating in other behavioral/ pharmacological trials, (iii) had significant cognitive impairment as indicated by the Abbreviated Mental Test [15] score $<6$ (sensitivity of 96\% and specificity of 94\%), (iv) and/or have other contraindication/severe comorbidities (e.g., severe hearing/vision impairment) that might limit their full participation in the study.

\section{Interventions}

For 4 weeks, the mHealth-delivered mindfulness yoga program was delivered to participants with biweekly 90-min training sessions through an enhanced online platform via Zoom, a video conferencing software. All training sessions were group-based and delivered by an intervener using an interactive, real-time approach. The program was tailored on the basis of the research team's previous experience in center-based mindfulness yoga intervention for PD $[6,7]$, which comprised the mindfulness practice of modified yoga sequences for PD and mindfulness meditation and breathing techniques. Each item was modified to target the functional impairments, such as turning, asymmetric body alignment, and facial stiffness, induced by PD motor symptoms. We encouraged all participants to perform daily 15 -min self-practice of mindful walking to deepen the mindfulness experience specific to balance, mobility, and FOG symptoms. Mindful walking is type of moving meditation, which can be practiced anywhere without restriction. During mindful walking, the participants were instructed to walk and shifts their weight and balance slowly, with conscious awareness of the body sensations and thoughts whilst walking. The theme of each session reflected one of the eight mindfulness attitudes: non-judgement, patience, beginner's mind, trust, non-striving, acceptance, letting go, and generosity [16]. The components of the mindfulness yoga program are presented in Table 1 . At the end of each session, the intervener facilitated mindfulness practice and experiences to consolidate learning and promote social interaction. The major components, including the same set of yoga sequences, mindful walking, mindfulness meditation, and breathing techniques (http://yoga. nursing.hku.hk/), of the previously tested mindfulness yoga program were revamped into a series of guided videos and stored on a webpage for self-practice to promote skill mastery. All participants were given personal access to the webpage. The daily self-practice of mindful walking was reinforced at the end of each class, and self-practice behaviors (i.e., frequency and time spent on self-practice) were queried via telephone and documented on a weekly basis.

\section{Online intervention delivery}

We invited potential participants to attend a face-to-face individual information session and assessment. Particularly, we reminded them to bring their personal mobile device for rehearsing the online conferencing procedures. An instruction manual summarizing home-based yoga setting and online conferencing procedures was provided with return demonstration. Participants were asked to self-prepare a yoga mat and yoga block. Before the commencement of the online intervention, an individual home-based Zoom session was conducted to ensure an appropriate setting (i.e., a yoga mat with a sturdy chair aside/next to a wall and supportive aids, such as a towel or thick mattress/yoga block). We took specific effort to identify the optimal placement of the smart device and the adjustment of camera's angle to capture the whole body of participant as much as possible, so that the intervener can respond to the participant's needs in real-time time. A group-based rehearsal session was also conducted on the day before the first session to ensure a smooth delivery of intervention.

\section{Safety and adverse events}

Safety is particularly important to minimize risks (e.g., fall risks) for patients with PD. All participants were instructed to perform the online yoga practice next to a wall beside a sturdy chair for additional support and with their caregiver(s) nearby if needed. In consideration of the fluctuating motor symptoms and physical functions of patients with PD, two research team members (including an experienced registered nurse in PD care) participated as observers throughout all online yoga sessions to ensure the participants' safety. If any risk of injury/ fall was observed/anticipated, the team would contact the participant via Zoom and/or telephone immediately to reinforce additional safety measures. All participants were instructed to inform the team if they encountered any implementation-related adverse events with medical referrals provided if indicated. Any adverse event would be documented and handled in accordance with the standard operating procedure.

\section{Data collection and outcome measures}

Data regarding sociodemographic characteristics (including age, gender, marital status, education, 
Table 1 Components of the mHealth-delivered, home-based mindfulness yoga program.

\begin{tabular}{|c|c|c|c|c|c|c|c|c|}
\hline Class & 1 & 2 & 3 & & 5 & 6 & & 8 \\
\hline Theme & Non-judging & Patience & $\begin{array}{l}\text { Beginner's } \\
\text { mind }\end{array}$ & Trust & Non-striving & Acceptance & Letting go & Generosity \\
\hline $\begin{array}{l}\text { Breathing techniques } \\
\text { (10 min) }\end{array}$ & \multicolumn{8}{|c|}{$\begin{array}{l}\text { Each class is started by greetings and introduction of each theme, followed by breathing exercises } \\
\text { Breathing exercises in easy pose: } \\
\text { - Bee breath/ Lion breath } \\
\text { - Cooling breath/ Alternate nostril breathing }\end{array}$} \\
\hline $\begin{array}{l}\text { Yoga sequence } \\
\text { (60 min) }\end{array}$ & \multicolumn{8}{|c|}{$\begin{array}{l}\text { Warm-up sequences: } \\
\text { - Easy pose with neck, shoulders, triceps, hands and wrists'stretch } \\
\text { - Easy twist } \\
\text { - Cat-cow stretch } \\
\text { - Child's pose } \\
\text { Sun salutations sequences, delivered in stepwise approach with modifications (12 poses): } \\
\text { - Mountain pose } \\
\text { - Upward salute pose } \\
\text { - Standing forward bend } \\
\text { - Lunge } \\
\text { - Plank pose } \\
\text { - Knees, chest and chin pose } \\
\text { - Cobra pose } \\
\text { - Downward-facing dog pose } \\
\text { - Lunge } \\
\text { - Standing forward bend } \\
\text { - Upward salute pose } \\
\text { - Mountain pose } \\
\text { Cool down sequences: } \\
\text { - Child pose } \\
\text { - Knee-to-chest } \\
\text { - Happy baby } \\
\text { - Corpse pose } \\
\text { - Side corpse pose and detailed instructions }\end{array}$} \\
\hline $\begin{array}{l}\text { Meditation } \\
\text { (15 min) }\end{array}$ & \multicolumn{8}{|c|}{$\begin{array}{l}\text { Meditation practice in corpse/easy pose: } \\
\text { - Body scan meditation (cultivation of awareness towards breath and body) } \\
\text { - Open-monitoring awareness meditation (cultivation of mindfulness towards mind and body) } \\
\text { - Loving-kindness meditation (cultivation of loving kindness to oneself and others) }\end{array}$} \\
\hline $\begin{array}{l}\text { Sharing and conclusion } \\
(5 \mathrm{~min})\end{array}$ & \multicolumn{8}{|c|}{$\begin{array}{l}\text { At the end of each session, the instructor would invite and encourage participants to share their experience, thoughts } \\
\text { and feelings of mindfulness yoga practice to promote social interaction, followed by concluding remarks to consolidate } \\
\text { learning and reinforce self-practice }\end{array}$} \\
\hline Daily self-practice & \multicolumn{8}{|c|}{ Daily 15-min self-practice of mindful walking, guided by pre-recorded video } \\
\hline Facilitation of self-practice & \multicolumn{8}{|c|}{$\begin{array}{l}\text { Guided videos of the same set of yoga sequences, mindful walking, mindfulness meditation, and breathing techniques } \\
\text { are accessible on http://yoga.nursing.hku.hk/ (Chinese version) to promote skill mastery and self-practice }\end{array}$} \\
\hline
\end{tabular}

employment, and income), clinical background (including year of onset, disease staging, and medication record) and the following outcomes were collected at baseline and 1-week post-intervention through Zoom interviews:

\section{Functional balance}

The primary outcome, functional balance was measured with the assessor-rated Berg and Balance Scale (BBS), which has been validated in people with PD [17]. BBS consists of 14 items that assess the balance performance of elderly adults in common everyday life activities on a five-point interval scale (0-4). This scale has a total score that ranges from 0 to 56 . A score between 0 and 20 indicates balance deficit, and scores of 21-40 and 41-56 represent acceptable balance and good balance, respectively.

\section{Severity of motor symptoms}

The severity of PD-related motor symptoms was measured with the validated Movement Disorder SocietyUnified Parkinson's Disease Rating Scale (MDS-UPDRS) (Chinese version) part III [18], which measures major PD motor symptoms, including tremors, rigidity, bradykinesia, gait, and postural instability. High scores indicate great disease severity. Motor examination was conducted by qualified personnel with a MDS-UPDRS certificate.

\section{Perceived balance confidence}

Perceived balance confidence was measured with the Activities-specific Balance Confidence (ABC) Scale (Chinese version) [19], which is a validated and reliable tool that is applied to measure the level of self-perceived confidence in balance from $0 \%$ (no confidence) to $100 \%$ 
(full confidence) in 16 indoor and outdoor activities. Participants were asked to report their perceived balance confidence during "on" (ABC-on) and "off" (ABC-off) states. $A$ high $A B C$ score indicates a high level of balance confidence.

\section{Perceived severity of freezing of gait}

FOG severity was measured with the validated FOG Questionnaire (Chinese version) (FOGQ) (Cronbach's alpha: 0.94) [20]. FOGQ is a self-reported questionnaire that consists of six items capturing FOG severity and gait. Each item is rated on a five-point interval scale that ranges from 0 , which indicates the absence of symptoms, to 4 , which indicates the most severe stage of symptoms. The total score of this scale ranges from 0 to 24 . A high score indicates severe FOG.

\section{Anxiety and depressive symptoms}

Anxiety and depressive symptoms was measured by applying the validated Hospital Anxiety and Depression Scale (HADS) (Chinese-Cantonese version), which is a self-reported questionnaire that consists of anxiety and depression subscales. Each subscale consists of seven items, and each item is rated on a fourpoint scale. A high score represents a high level of psychological distress. HADS has been suggested for use in the PD population because somatic symptoms that may potentially overlap with Parkinsonian manifestations are not assessed with this scale [21]. Moreover, it focuses on measuring the negative emotions of anxiety and depression, which have been reported as being the most prominent psychological factors in patients with PD.

\section{Mindfulness}

Mindfulness level was measured with the 20-item validated short-form Five-Facet Mindfulness Questionnaire (Chinese version) [22]. Five key aspects of mindfulness, including observing, describing, acting with awareness, non-judgment of inner experience, and non-reaction to inner experience, were assessed by using a five-point Likert scale. A high score indicates a high level of mindfulness at present.

\section{HRQOL}

HRQOL was assessed by using the validated Parkinson's Disease Questionnaire-8 (Chinese version) [23, 24]. This eight-item scale provides a summary index score that captures disease-specific HRQOL regarding mobility, daily living activities, emotional wellbeing, social support, cognitions, communication, bodily discomfort, and stigma. A high score indicates poor HRQOL.

\section{Treatment fidelity}

For treatment fidelity, exercise intensity in terms of perceived exertion was measured by using the Borg Category-Ratio 10 (CR10) scale at the end of each class. CR10 is a validated tool that is used to quantify an individual's perception of physical demands during physical work $[25,26]$. A CR score ranging from 0 (nothing at all) to 10 (extremely strong) indicates exercise intensity. A score of 4 (somewhat hard) to 7 (very hard) indicates the safe, moderate-to-high intensity of exertion related to the physical workout [27]. Treatment fidelity was assessed by means of virtual 'on-site' observation by the first author with the use of a pre-constructed observation checklist.

\section{Qualitative exploration}

Individual semi-structured interviews were conducted to explore the experience of patients with PD in practicing mindfulness yoga through the home-based, mHealthdelivered approach with a focus on conditions that influenced the participants' motivation, acceptability, real-life practice, and area for improvement. Another area of focus was the participants' perceived effects of the overall and individual components of the intervention and how and why they work or do not work.

\section{Statistical analysis}

Sociodemographic characteristics and clinical background were summarized using descriptive analyses. Means and SDs were used to describe normally distributed continuous variables, whereas medians and IQRs were used for continuous variables that were not normally distributed. Proportions were used for categorical variables. Pre- and post-intervention comparisons of outcomes between variables at the baseline and the 1-week follow-up were conducted. Paired Student $t$-tests were performed for normally distributed continuous variables, Wilcoxon signed-rank tests were conducted for nonnormally distributed variables, and chi-square tests were applied for categorical variables. The analyses were performed by using IBM SPSS version 25 .

For qualitative data, audio-taped interviews were transcribed verbatim and managed by using NVivo. Two experienced qualitative researchers inductively analysed the transcribed interviews with a thematic analysis approach [28]. The coded units were sorted into categories and subcategories and analyzed for recurrent themes and patterns. Emerging categories were reviewed for resonance with the quantitative findings. Such qualitative findings served a complementary purpose in reaching conclusions on the preliminary effects and acceptability 
of home-based, mHealth-delivered mindfulness yoga practice for PD rehabilitation.

\section{Results}

\section{Patient engagement}

Figure 1 depicts the study flow. With the COVID-19 outbreak, all out-patient services were kept minimal. Among the ten potential participants screened, all met the eligibility criteria and one declined to participate (enrolment rate: $80 \%)$ due to unavailability and another declined because of mHealth illiteracy. Figure 2 shows the screen capture of one intervention session. The mean and standard deviation (SD) attendance rates were 7.88 (0.33) sessions with a participation rate of $98.4 \%$. One participant did not attend one session due to a schedule conflict with a medical appointment. All participants attended the online follow-up assessment sessions. The compliance rates for the daily 15 -min mindful walking self-practice during the intervention period were $100 \%$. The mean (SD) frequencies of mindful walking were 1.8 (0.2) times per day. No serious adverse event was observed in any of the participants.

\section{Patients' characteristics}

The participants' sociodemographic and clinical characteristics are summarized in Table 2. The mean age of participants was 63.1 years (SD 5.4 years), and half of the participants were male. The majority of the patients were married (62.5\%) and received tertiary education (62.5\%). All were living with a spouse/family, and half received social security allowance. All had moderate PD (Hoehn and Yahr scale, stage 3). Their mean (SD) levodopa equivalent dose was 738.4 (469.2).

\section{Tele-assessment of outcome measures}

All outcomes were assessed uneventfully using Zoom. Participants were familiar with videoconferencing procedures and followed commands of physical examination. Table 3 presents the scores of all outcome variables at baseline and 1-week follow-up.

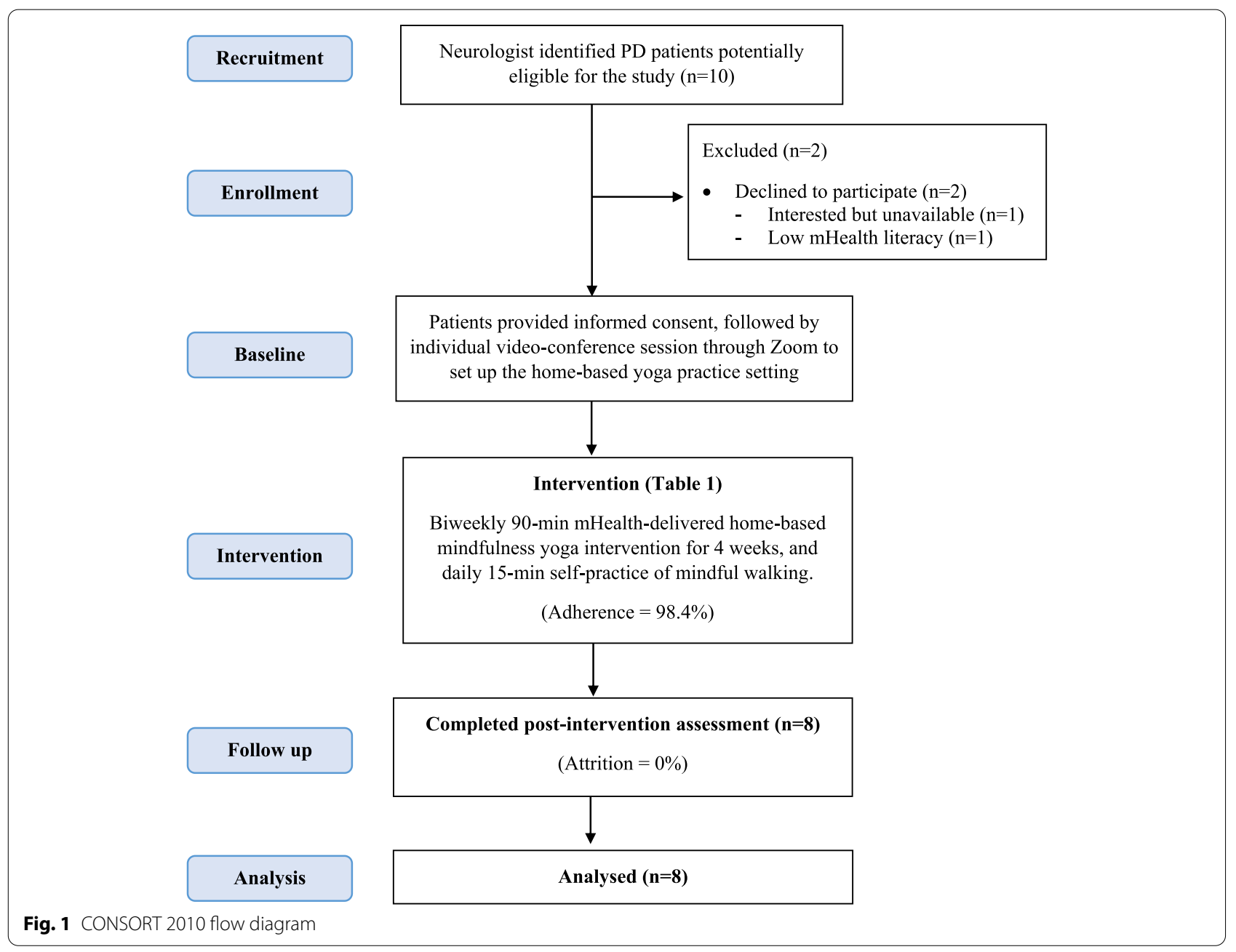




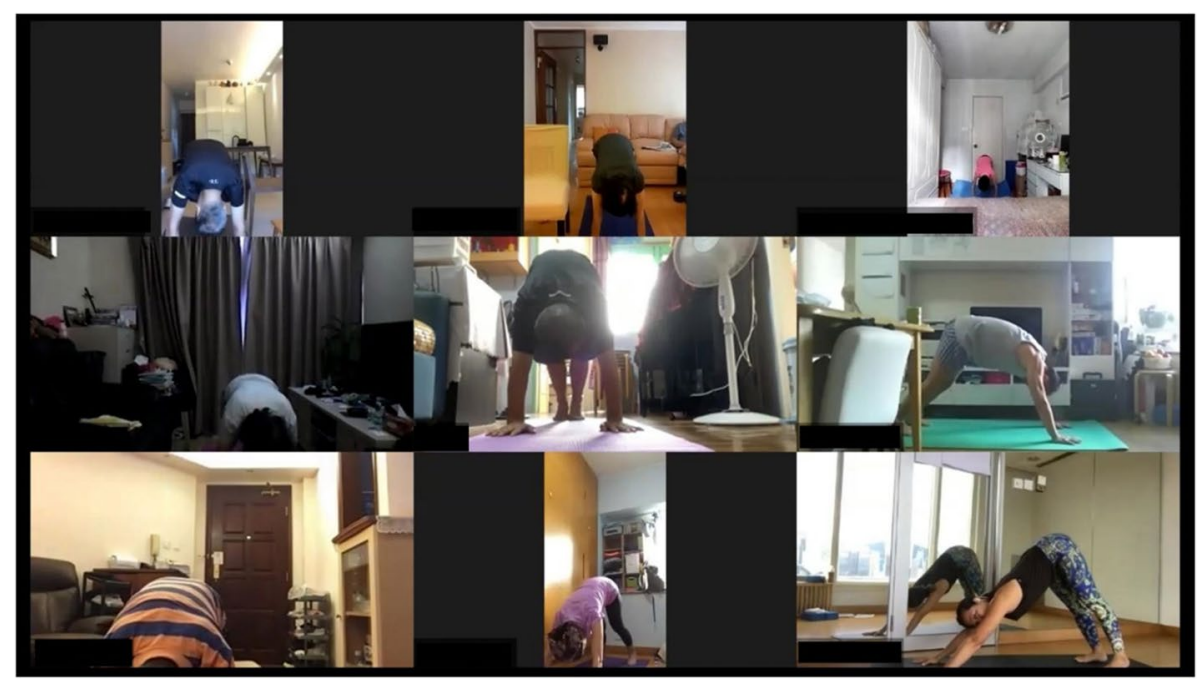

Fig. 2 Screen capture of the intervention session (8 participants and 1 instructor, consent obtained)

Table 2 Baseline sociodemographic and clinical characteristics of the participants

\begin{tabular}{ll}
\hline Characteristics & $\begin{array}{l}\text { Participants }(\boldsymbol{n}=\mathbf{8}), \\
\text { mean (SD) or no. } \\
\text { (\%) }\end{array}$ \\
\hline $\begin{array}{l}\text { Age } \\
\text { Gender }\end{array}$ & $63.1(5.4)$ \\
Male & $4(50.0)$ \\
Female & $4(50.0)$ \\
Marital Status & \\
Single, widowed & $3(37.5)$ \\
Married & $5(62.5)$ \\
No. of children & $1.6(0.7)$ \\
Educational level & \\
Secondary & $3(37.5)$ \\
Tertiary & $5(62.5)$ \\
Living with spouse or family & $8(100)$ \\
Social security allowance & $4(50)$ \\
Hoehn and Yahr stage & $3(0)$ \\
Levodopa equivalent dose & $738.38(469.2)$ \\
\hline
\end{tabular}

\section{Physical outcomes}

Participants showed a significant improvement in functional balance (BBS: mean change $=5.13 ; p<0.001$; Cohen's $\mathrm{d}=2.84$ ) and overall motor symptoms (MDSUPDRS-III: mean change $=12.63 ; P=0.002$; Cohen's $\mathrm{d}=2.33$ ) from baseline to 1 -week follow-up. For subjective measures, perceived balance confidence during the "on" (ABC-ON: mean change $=3.63 ; P=0.28)$ and "off" states (ABC-OFF: mean change $=-13.22 ; P=0.14$ ) and perceived FOG (FOGQ: mean change $=1.38$; $P=0.23$ ) were insignificant.

\section{Mental health and HRQOL outcomes}

For psychological distress, participants showed a significant reduction in anxiety (HADS-anxiety: mean change $=9.00 ; P=0.002$; Cohen's $d=1.73$ ) and depressive symptoms (HADS-depression: mean change $=3.63$; $P=0.04$; Cohen's $\mathrm{d}=0.91$ ). For mindfulness, an improving trend was noted regarding the domain of "nonjudging to inner experience" (FFMQ-non-judging: mean change $=-2.25 ; P=0.05)$. HRQOL showed insignificant improvement (PDQ-8: mean change $=0.78 ; P=0.82$ ).

\section{Feasibility, treatment intensity and safety of the mindfulness yoga programme}

We recruited 10 eligible participants from the neurology out-patient clinic, with an enrolment rate of $80 \%$. The adherence rate of online intervention was $98.4 \%$, in which one participant missed a class due to a schedule clash with medical appointment. The mean (SD) of the Borg CR10 was 5.68 (1.03) with a range of 4 to 8 , indicating the safety and moderate intensity of the exercise. All online sessions demonstrated a satisfactory treatment fidelity. The self-practice of mindful walking had a mean (SD) time of 23.13 (12.64) minutes per day and ranged from 10 to $60 \mathrm{~min}$ per day. All participants attended the pre- and post-intervention follow-ups. For safety concerns, one participant exhibited instability due to dyskinesia during the first virtual class on yoga practice. Our team immediately contacted the participant through Zoom and reinforced safety precautions, including practicing next to a wall and placing a chair next to the yoga mat for support. 
Table 3 Results of the outcome variables $(n=8)$

\begin{tabular}{|c|c|c|c|c|}
\hline Outcome & T0, Mean (SD) & T1, Mean (SD) & Change $(95 \% \mathrm{Cl})$ & $P$ Value \\
\hline$A B C-O N \S$ & $87.07(10.56)$ & $85.30(10.27)$ & $3.63(-3.73$ to 11.00$)$ & .28 \\
\hline ABC-OFF§ & $61.72(13.55)$ & $74.94(18.64)$ & $-13.22(-32.09$ to 5.65$)$ & .14 \\
\hline BBS $\S$ & $50.00(2.20)$ & $55.13(1.73)$ & $-5.13(-6.61$ to -3.61$)$ & $<.001$ \\
\hline MDS-UPDRS II* & $25.25(5.90)$ & $12.63(4.50)$ & 12.63 (6.52 to 18.73$)$ & .002 \\
\hline FOGQ & $12.38(5.04)$ & $11.00(4.24)$ & $1.38(-1.11$ to 3.86$)$ & .23 \\
\hline HADS-anxiety & $12.75(2.77)$ & $3.75(2.82)$ & 9.00 (4.64 to 13.36$)$ & .002 \\
\hline HADS-depression & $8.88(2.03)$ & $5.25(3.73)$ & 3.63 (0.31 to 6.94$)$ & .04 \\
\hline FFMQ-Total & & & & .82 \\
\hline FFMQ-Observing & $14.13(4.26)$ & $13.38(3.66)$ & $0.75(-1.43$ to 2.93$)$ & .44 \\
\hline FFMQ-Describing & $16.50(2.14)$ & $14.13(3.18)$ & $2.38(-1.17$ to 5.92$)$ & .16 \\
\hline FFMQ-Acting with awareness & $14.37(3.62)$ & $15.13(2.23)$ & $-0.75(-3.46$ to 1.96$)$ & .53 \\
\hline FFMQ-Non-judgment & $10.13(3.60)$ & $12.38(2.00)$ & $-2.25(-4.56$ to 0.06$)$ & .055 \\
\hline FFMQ-Non-reaction & $15.38(2.13)$ & $14.75(1.39)$ & 0.63 (-1.47 to 2.72$)$ & .50 \\
\hline PDQ-8 summary index & $24.22(14.05)$ & $23.44(11.93)$ & 0.78 (-6.96 to 8.53$)$ & .82 \\
\hline
\end{tabular}

Abbreviations ABC, Activities-Specific Balance Confidence Scale percentage confidence; on, on medication state; off, off medication state, BBS, Berg Balance Scale, FFMQ, Five Facet Mindfulness Questionnaire; FOGQ, Freezing of Gait Questionnaire, HADS, Hospital Anxiety and Depression Scale, MDS-UPDRS III, Movement Disorders Society United Parkinson's Disease Rating Scale, Part III (excluded item 3.3 and 3.12 due to virtual assessment), PDQ-8, 8-item Parkinson's Disease Questionnaire. All assessments were conducted virtually via Zoom at baseline (T0) and immediately post-intervention ( $\mathrm{T} 1)^{\S}{ }^{\S}$ an negative change score indicates improvement

Overall, all classes were delivered uneventfully without an adverse event.

\section{Qualitative feedback}

Participants reported that they had positive experience with the intervention. They identified several merits of the intervention as follows:

The mindfulness yoga program was interactive and interesting, thus greatly increasing their motivation in establishing sustained practice. The participants requested additional online sessions and verbalized remarkable commitment and interest in attending longterm mHealth-delivered home-based mindfulness yoga for rehabilitation.

"I did not expect exercising at home could be so enjoyable. It was really fun to practice in a group virtually, and the interactive real-time approach using Zoom really kept us motivated. I want to have more of it." (Participant 2)

The home-based approach was convenient and reassuring, which reduced the burden associated with transportation.

"I used to spend over an hour in travelling to attend supervised exercise classes. Now, it's convenient that we can practice at home. I can easily integrate it (the program) into my daily routine without spending extra time and money on transportation."(Participant 1)
Moreover, the participants' adherence would not be hindered by common barriers to participation in centerbased programs, such as adverse weather, the recent public health measures against COVID-19, and safety concerns related to social movement activities in the local community.

"I worried about going out since the start of the social movements, and it's now even worse due to the COVID-19 outbreak. I felt like being trapped at home and I was much stiffer. It's so great that I could be active again (by joining this program) even at home."(Participant 5)

The participants also commented that the home-based yoga setup via Zoom was manageable because it required only a yoga mat, chair, and one smart device installed with Zoom. The assistance from caregiver/families is deemed helpful to facilitate the set up.

"Although I am not very familiar with computer and Zoom, it's quite easy with the demonstration and return demonstration (by the research team), and my husband helped me with that." (Participant 4)

They verbalized concerns about the execution of yoga postures and suggested that ideally, one to two fundamental in-person yoga classes should be provided before virtual classes to enhance skill mastery.

"Although the instructor taught step-by-step and I can follow the yoga sequences, I am not sure if I fol- 
lowed the steps correctly. It would be ideal if there's one to two in-person classes beforehand, but I understand that it might not be feasible during the COVID-19." (Participant 3)

The overall comments on the intervention effects covered a range of physical and mental benefits including improved mood and sleep and enhanced calmness, balance, mobility, and gait. Regarding intervention components, all participants reported that the mindful walking practice was associated with improved walking confidence, improved gait control during gait initiation and walking, and reduced FOG episodes. Such benefits were particularly apparent during the "off" state.

"During the mindful walking, I felt like I learned how to walk again, and my mind was reconnected to my legs and feet. The overall program really helped my body coordination, especially during my off-time, I was less anxious and could function better." (Participant 5)

The participants felt calm and relaxed after practicing breathing exercises, and almost $40 \%$ reported that meditation practices were associated with improved sleeping quality and emotional stability.

\section{Discussion}

To the best of our knowledge, this prospective pilot study is the first attempt to test the feasibility of a home-based interactive mindfulness yoga intervention through an online platform to help promote the physio-psychospiritual well-being of patients with PD. The findings of this study indicated that the mHealth-delivered homebased mindfulness yoga programme was feasible and safe without adverse effects, and particularly well received for patients with PD during the COVID-19 pandemic.

For people with PD, adhering to rehabilitation programs is particularly crucial and becomes challenging as they experience progressive motor dysfunctions and mental complications over the course of their illness. Patients with PD begin to experience motor fluctuations, including wearing-off effects and 'on-off' phenomena, with increasing frequency after $2-5$ years on average due to the short and/or unpredictable response time of longterm levodopa use [29]. All these complications considerably affect their functional performance, planned schedule, motivation, and adherence to participation in center-based programs. A 2016 systematic review of mind-body exercises reported that an increase in the intervention frequency may be associated with unfavorable enrolment and adherence among people with PD [5]. Previous studies on center-based mind-body exercise interventions with a weekly frequency reported that the adherence rates of participants with PD range from $56.4 \%$ to $92.9 \%$ [7, 30-32]. Our pilot findings demonstrated that the biweekly mindfulness yoga program was remarkably well-received among patients with PD despite its increased frequency as evidenced by its high enrolment and adherence rates of $80 \%$ and $98.4 \%$, respectively. This finding highlighted the particular relevance and importance of the mHealth-delivered home-based approach to PD rehabilitation. Such an approach has low resource demands and removes the common external barriers to participation in center-based programs [33]. These barriers include adverse weather events, the social distancing and public health measures used to combat the COVID-19 pandemic, and safety concerns related to social movement activities in the local community. In addition, delivering yoga in a familiar and controlled home setting may facilitate the patients' adherence and sustained engagement in frequent yoga practice.

Previous evidence showed that the high frequency of yoga practice is associated with low emotional and physiological reactivity, high emotional stability, and resilience to negative stimuli or vulnerability $[34,35]$, as well as increased physical health benefits among older adults [36]. More frequent yoga practice could deepen one's mind-body awareness and connections [37]. However, its effects on cultivating mindfulness attitudes remained unknown. The virtual delivery of yoga practice may affect the immersion of participants in "in-moment" experiences and may compromise the quality of presentmoment awareness and thus the cultivation of mindfulness. As such, the intervention effect may be mediated through the psychosocial effects of virtual gatherings or the physiological effects of exercise rather than the cultivation of mindfulness attitudes. Hence, future trials should adopt an active control group for comparison and investigate the mediating roles of physical performance and mindfulness level in the effect of mindfulness training on psychological distress and HRQOL, as well as strategies optimizing the "in-moment" experiences of virtually delivered mindfulness yoga practice. Mocanu and Mohr [34] found that long-term yoga practice is linked with high "moment-to-moment" consciousness and considerable improvement in clinical symptoms and life satisfaction. Hence, we suggest prolonging the duration of future mind-body interventions to achieve subjective improvement. In corroboration with previous studies on mind-body exercises for PD [5, 7], delivering interventions through biweekly dosages for $8-10$ weeks is essential to enhance the skill mastery of mindfulness yoga and promote physio-psycho-spiritual gains, as well as ensure adequate power and time to translate objective functional improvements into subjective positive experience that can be perceived by patients in daily living. 
Corroborating with a recent report of PD management during the COVID-19 pandemic [38], the findings of the present study also supported the implementation of telemedicine to assess physical symptoms of PD. Indeed, previous studies [39, 40] have demonstrated that most physical symptoms can be visualized and many core features of PD, except rigidity and postural reflexes, can be captured and rated through videoconference. This pilot study has further demonstrated the feasibility of tele-assessment of physical symptoms in local setting, given the fact that a stable bandwidth is essential to ensure an optimal video quality in terms of resolution and smoothness of motion. Although there is some loss of information when using tele-approach to rate BBS/ MDS-UPDRS-III compared to in-person ratings, such tele-assessment approach may serve as a low-cost and convenient alternative to facilitate the continuum of PD care in the community.

Last but not least, patient qualitative data indicated that mindful walking was particularly helpful in improving gait control and FOG symptoms, particularly during wearing-off. The high compliance of mindful walking self-practice suggests participants' high acceptance to this complementary meditative practice, which can be practiced anywhere without any equipment or restrictions. Indeed, managing FOG is difficult owing to its multifaceted pathophysiology, which involves an interplay between motor elements (dysregulated stepping mechanisms) and non-motor elements (anxiety and cognitive decline) [2]. Most research to date has examined the effects of center-based motor skill training combined with external cueing strategies (i.e., rhythmic auditor stimulation) for FOG management [41, 42]. However, such modalities cannot be implemented in the form of ambulatory strategies to assist patients in their daily activities regardless of their location. The self-practice and internalizing nature of mindful walking techniques can potentially be adopted as an ambulatory strategy that can readily be integrated into daily living to enhance body coordination and alleviate FOG symptoms. Mindfulness techniques may serve as an internal cueing strategy for mental activation to enhance "in-present" mind-body awareness and coordination, which present promising therapeutic potential for PD rehabilitation. The incorporation of mindfulness and motor skill training may provide additional avenues by which a person can improve mind-body coordination for FOG management. Its potential clinical values should be further evaluated by using a methodological design with increased rigor, i.e., a randomized controlled design with a large sample size.

\section{Implications}

The home-based mindfulness yoga intervention, which was delivered interactively via an online platform and utilized prerecorded videos for self-practice, gave a unique opportunity to provide much-needed mind-body rehabilitation to patients with PD for the likely improvement of functional balance, motor symptoms, and mental status. This feasibility study showed that the mHealthdelivered home-based approach is a feasible, safe, and well-received option for patients with PD, in particularly during the COVID-19 pandemic. The experience of this study might aid the development of large randomized controlled trials or similar programs for patients with PD or other neurological conditions, especially those with physical impairment or limited resources who are unable to reach center-based rehabilitation programs frequently. Moreover, it appeared to be particularly helpful and accessible during times of infectious disease outbreaks. We recommend increasing the intervention training sessions to twice a week for 8-10 weeks to enhance treatment effects, especially effects on the component of subjective functional outcomes and HRQOL. The effects of mindful walking should be further evaluated in future trials for FOG management.

\section{Limitations}

Despite its positive outcomes, this study had several limitations. First, the sample size was small and a comparison group was not established; thus, caution is needed when interpreting its findings. Second, some features of PD, including rigidity and postural reflexes, cannot be captured and assessed on videoconference. Third, adherence to mindful walking was self-reported and thus might be overestimated owing to social desirability bias and recall bias. Future studies should adopt a large sample size and preferably use a randomized controlled trial design. The subjective experience of the participants should also be explored by collecting qualitative feedback throughout the study, and the sustained effects of the intervention should be examined with a lengthy follow-up.

\section{Conclusions}

This study provided evidence supporting the feasibility of the mHealth-delivered home-based mindfulness yoga program to people with mild-to-moderate PD. Although motor dysfunctions and PD pathology have been identified as factors that might challenge the feasibility, safety, and acceptability of virtual rehabilitation in this vulnerable group, this pilot study showed that the mHealthdelivered home-based mindfulness yoga intervention was feasible, safe and well-accepted among people with $\mathrm{PD}$, which was particularly helpful and accessible during 
times of infectious disease outbreaks. Future studies with enhanced rigor and increased sample size are needed to establish efficacy in patients with PD and those with other neurological conditions or physical impairments.

\section{Acknowledgements}

We would like to extend our sincere gratitude and appreciation all the participants for their active engagement and support of this study. We were also grateful to the research assistant, Mr. Leo Chun Chung Lam, for his dedicated assistance and contribution to this research paper.

\section{Authors' contributions}

Dr. Kwok had full access to all the data in the study and takes responsibility for the integrity of the data and the accuracy of the data analysis., Study concept and design, Kwok, Auyeung, Acquisition analysis, or interpretation of data, Kwok, Lee, Choi, Chau, Auyueng, Drafting of the manuscript, Kwok, Critical revision of the manuscript for important intellectual content, Kwok, Lee, Choi, Chau, Auyueng, Statistical analysis, Kwok, Chau, Obtained funding, Kwok Administrative, technical, or material support, Kwok, Auyeung, Study supervision, Auyeung. The authors read and approved the final manuscript.

\section{Funding}

Research reported in this article was supported by the Seed Fund for Basic Research, the University of Hong Kong.

\section{Availability of data and materials}

The datasets generated and/or analysed during the current study are not publicly available but are available from the corresponding author on reasonable request.

\section{Declarations}

\section{Ethics approval and consent to participate}

This study was performed in line with the principles of the Declaration of Helsinki. Ethics approval has been obtained from the ethics committee of the Institutional Review Board of the University of Hong Kong/Hospital Authority Hong Kong West Cluster (HKU/HA HKW IRB) (Reference number: UW 19-535). Written informed consent was obtained from all the participants.

\section{Consent for publication}

All participants has given informed consent for publication of potentially identifying images in an online open-access publication.

\section{Competing interests}

None reported. No competing interests. Dr. Jojo Kwok would like to declare her role of Editorial Board Member of BMC Public Health (from July 2021-present).

\section{Author details}

${ }^{1}$ School of Nursing, Li Ka Shing Faculty of Medicine, The University of Hong Kong, Hong Kong 4/F, William MW Mong Block, 21 Sassoon Road, Pokfulam, Hong Kong SAR, China. ${ }^{2}$ Department of Medicine, Pamela Youde Nethersole Eastern Hospital, Chai Wan, Hong Kong SAR, China.

Received: 22 March 2021 Accepted: 20 January 2022

Published online: 07 February 2022

\section{References}

1. Nonnekes J, Bereau M, Bloem BR. Freezing of gait and its levodopa paradox. JAMA Neurol. 2020;77(3):287-8.

2. Gilat $\mathrm{M}$, et al. Freezing of gait: Promising avenues for future treatment. Parkinsonism Relat Disord. 2018;52:7-16

3. Kwok JYY, Huang TW, Tretriluxana J, Auyeung M, Chau PH, Lin CC, Chan HYL. Symptom Burden and Unmet Support Needs of Patients With Parkinson's Disease: a Cross-Sectional Study in Asia-Pacific Regions. J Am Med Dir Assoc. 2021;22(6):1255-64. https://doi.org/10.1016/j.jamda.2020. 09.012 .
4. Domingos J, et al. The European Physiotherapy Guideline for Parkinson's Disease: Implications for Neurologists. J Parkinsons Dis. 2018;8(4):499-502.

5. Kwok JYY, Choi KC, Chan HYL. Effects of mind-body exercises on the physiological and psychosocial well-being of individuals with Parkinson's disease: A systematic review and meta-analysis. Complement Ther Med. 2016;29:121-31.

6. Kwok JYY, et al. The effects of yoga versus stretching and resistance training exercises on psychological distress for people with mild-to-moderate Parkinson's disease: study protocol for a randomized controlled trial. Trials. 2017;18(1):509.

7. Kwok JYY, et al. The effects of mindfulness yoga versus stretching and resistance training exercises on anxiety and depression for people with Parkinson's disease: a randomized clinical trial. JAMA Neurol. 2019;76(7):755-63.

8. Duncan RP, Earhart GM. Randomized controlled trial of community-based dancing to modify disease progression in Parkinson disease. Neurorehabil Neural Repair. 2012;26(2):132-43.

9. Ellingson LD, Zaman A, Stegemöller EL. Sedentary Behavior and Quality of Life in Individuals With Parkinson's Disease. Neurorehabil Neural Repair. 2019;33(8):595-601.

10. Puccinelli PJ, da Costa TS, Seffrin A, de Lira CAB, Vancini RL, Nikolaidis PT, Knechtle B, Rosemann T, Hill L, Andrade MS. Reduced level of physical activity during COVID-19 pandemic is associated with depression and anxiety levels: an internet-based survey. BMC Public Health. 2021;21(1):425. https://doi.org/10.1186/s12889-021-10470-z.

11. Lee JJ, Tsang WN, Yang SC, Kwok JYY, Lou VWQ, Lau KK. Qualitative Study of Chinese Stroke Caregivers' Caregiving Experience During the COVID-19 Pandemic. Stroke. 2021;52(4):1407-14. https://doi.org/10.1161/STROK EAHA.120.032250.

12. Lee J-A, et al. Effective behavioral intervention strategies using mobile health applications for chronic disease management: a systematic review. BMC Med Inform Decis Mak. 2018;18(1):12.

13. Mathersul DC, Mahoney LA, Bayley PJ. Tele-yoga for Chronic Pain: Current status and future directions. Global advances in health and medicine. 2018;7:2164956118766011.

14. More P, et al. Development, validation, and feasibility of a generic yogabased intervention for Generalized Anxiety Disorder. Complement Ther Med. 2021:63:102776.

15. Chu, L., et al., Validation of the Abbreviated Mental Test (Hong Kong version) in the elderly medical patient. 1995.

16. Kabat-Zinn, J. and T.N. Hanh, Full catastrophe living: Using the wisdom of your body and mind to face stress, pain, and illness. 2009: Delta.

17. Scalzo PL, et al. Validation of the Brazilian version of the Berg balance scale for patients with Parkinson's disease. Arq Neuropsiquiatr. 2009;67(3B):831-5.

18. Goetz CG, et al. Movement Disorder Society-Sponsored Revision of the Unified Parkinson's Disease Rating Scale (MDS-UPDRS): Scale Presentation and Clinimetric Testing Results. Mov Disord. 2008;23(15):2129-70.

19. Mak MK, et al. Validation of the Chinese translated activities-specific balance confidence scale. Arch Phys Med Rehabil. 2007;88(4):496-503.

20. Ou R, et al. Freezing of gait in Chinese patients with Parkinson disease. J Neurol Sci. 2014;345(1-2):56-60.

21. Rodriguez-Blazquez C, et al. Psychometric attributes of the Hospital Anxiety and Depression Scale in Parkinson's disease. Mov Disord. 2009:24(4):519-25.

22. Hou J, et al. Validation of a Chinese version of the Five Facet Mindfulness Questionnaire in Hong Kong and development of a short form. Assessment. 2014;21(3):363-71.

23. Jenkinson C, et al. The Parkinson's Disease Questionnaire (PDQ-39): development and validation of a Parkinson's disease summary index score. Age Ageing. 1997;26(5):353-7.

24. Tsang KL, et al. Translation and validation of the standard Chinese version of PDQ-39: a quality-of-life measure for patients with Parkinson's disease. Mov Disord. 2002;17(5):1036-40.

25. Borg, G.A., Psychophysical bases of perceived exertion. Medicine \& science in sports \& exercise, 1982.

26. Borg, G., Borg's perceived exertion and pain scales. 1998: Human kinetics

27. Borg G. Psychophysical scaling with applications in physical work and the perception of exertion. Scand J Work Environ Health. 1990;16(Suppl 1):55-8. 
28. Braun V, Clarke V. Using thematic analysis in psychology. Qual Res Psychol. 2006;3(2):77-101.

29. Freitas ME, Hess CW, Fox SH. Motor Complications of Dopaminergic Medications in Parkinson's Disease. Semin Neurol. 2017;37(2):147-57.

30. Foster ER, et al. Community-Based Argentine Tango Dance Program Is Associated With Increased Activity Participation Among Individuals With Parkinson's Disease. Arch Phys Med Rehabil. 2013;94(2):240-9.

31. Colgrove YS, et al. Effect of yoga on motor function in people with Parkinson's disease: a randomized, controlled pilot study. J Yoga Phys Ther. 2012;2(2):112.

32. Ni M, et al. Comparative effect of power training and high-speed yoga on motor function in older patients with Parkinson disease. Archives of physical medicine and rehabilitation. 2016;97(3):345-54 (e15).

33. Cheng C, et al. Development and Feasibility of a Mobile Health-Supported Comprehensive Intervention Model (CIMmH) for Improving the Quality of Life of Patients With Esophageal Cancer After Esophagectomy Prospective, Single-Arm, Nonrandomized Pilot Study. J Med Internet Res. 2020;22(8):e18946.

34. Mocanu E, et al. Reasons, years and frequency of yoga practice: Effect on emotion response reactivity. Front Hum Neurosci. 2018;12:264.

35. Kwok JYY, Choi EPH, Chau PH, Wong JYH, Fong DYT, Auyeung M. Effects of spiritual resilience on psychological distress and health-related quality of life in Chinese people with Parkinson's disease. Qual Life Res. 2020;29(11):3065-73. https://doi.org/10.1007/s11136-020-02562-x.

36. Roland KP, Jakobi JM, Jones GR. Does yoga engender fitness in older adults? A critical review. J Aging Phys Act. 2011;19(1):62-79.

37. Shapiro, S.L. and L.E. Carlson, The art and science of mindfulness: Integrating mindfulness into psychology and the helping professions. 2009: American Psychological Association.

38. Papa SM, et al. Impact of the COVID-19 Pandemic on Parkinson's Disease and Movement Disorders. Mov Disord. 2020;35(5):711-5.

39. Venkataraman K, et al. Tele-Assessment of the Berg Balance Scale: Effects of Transmission Characteristics. Arch Phys Med Rehabil. 2017;98(4):659664.e1.

40. Flynn A, et al. Home-based exercise monitored with telehealth is feasible and acceptable compared to centre-based exercise in Parkinson's disease: A randomised pilot study. Clin Rehabil. 2021;35(5):728-39.

41. Medijainen K, et al. Versatile guideline-based physiotherapy intervention in groups to improve gait speed in Parkinson's disease patients. NeuroRehabilitation. 2019;44(4):579-86.

42. Cassimatis C, et al. The effectiveness of external sensory cues in improving functional performance in individuals with Parkinson's disease: a systematic review with meta-analysis. Int J Rehabil Res. 2016;39(3):211-8.

\section{Publisher's Note}

Springer Nature remains neutral with regard to jurisdictional claims in published maps and institutional affiliations.

Ready to submit your research? Choose BMC and benefit from:

- fast, convenient online submission

- thorough peer review by experienced researchers in your field

- rapid publication on acceptance

- support for research data, including large and complex data types

- gold Open Access which fosters wider collaboration and increased citations

- maximum visibility for your research: over 100M website views per year

At BMC, research is always in progress.

Learn more biomedcentral.com/submissions 